\title{
Combining transcranial ultrasound with intelligent communications methods to enhance the remote assessment and management of stroke patients - Framework for a technology demonstrator
}

\author{
Alasdair Mort ${ }^{1}$, Leila Eadie ${ }^{1}$, Luke Regan ${ }^{2,3}$, Ashish Macaden ${ }^{1,3}$, David Heaney ${ }^{1}$, Matt- \\ Mouley Bouamrane ${ }^{1}$, Gordon Rushworth ${ }^{3,4}$, Philip Wilson ${ }^{1}$ \\ ${ }^{1}$ Centre for Rural Health, University of Aberdeen, Centre for Health Science, Old Perth Road, \\ Inverness, Scotland, U.K. \\ ${ }^{2}$ Highland Medical Education Centre, University of Aberdeen, Centre for Health Science, \\ Old Perth Road, Inverness, Scotland, U.K. \\ ${ }^{3}$ Raigmore Hospital, Old Perth Road, Inverness, NHS Highland, Scotland, U.K. \\ ${ }^{4}$ Highland Clinical Research Facility, Centre for Health Science, Inverness, Scotland, U.K.
}

\section{Corresponding author:}

Dr Alasdair Mort, Centre for Rural Health, University of Aberdeen, Centre for Health Science, Old Perth Road, Inverness, IV2 3JH, Scotland, UK

E-mail: a.mort@abdn.ac.uk

Telephone: +0044 (0)1463255886 


\begin{abstract}
With over 150,000 strokes in the UK every year, and more than 1 million living survivors, stroke is the third most common cause of death and the leading cause of severe physical disability among adults. A major challenge in administering timely treatment is determining whether the stroke is due to vascular blockage (ischaemic) or haemorrhage. For patients with ischemic stroke, thrombolysis (i.e. pharmacological 'clot-busting') can improve outcomes when delivered swiftly after onset and current NHS Quality Improvement Scotland guidelines are for thrombolytic therapy to be provided to at least $80 \%$ of eligible patients within 60 minutes of arrival at hospital. Thrombolysis in haemorrhagic stroke could severely compound the brain damage so administration of thrombolytic therapy currently requires near immediate care in a hospital, rapid consultation with a physician and access to imaging services (X-ray computed tomography (CT) or magnetic resonance imaging) and intensive care services. This is near impossible in remote and rural areas, and stroke mortality rates in Scotland are 50\% higher than in London. We here describe our current project developing a technology demonstrator with ultrasound imaging linked to an intelligent, multi-channel communications device - connecting to multiple $2 \mathrm{G} / 3 \mathrm{G} / 4 \mathrm{G}$ networks and/or satellites - in order to stream live ultrasound images, video and two-way audio streams to hospital-based specialists who can guide and advise ambulance clinicians regarding diagnosis. With portable ultrasound machines located in ambulances or general practices, use of such technology is not confined to stroke, although this is our current focus. Ultrasound assessment is useful in many other immediate care situations, suggesting potential wider applicability for this remote support system. Although our research programme is driven by rural need, the ideas are potentially applicable to urban areas where access to imaging and definitive treatment can be restricted by a range of operational factors.
\end{abstract}

\title{
Keywords:
}

Acute Stroke Emergency Treatment, Medical Data and Image Transfer in Remote Locations, Technology-Supported Diagnosis 


\section{Introduction}

\section{The burden of stroke and the assessment and treatment of stroke in the acute phase}

Around 150,000 people in the United Kingdom (UK) have a stroke each year, costing between $£ 3.5$ and $£ 8$ billion, which includes formal and informal care provision, and lost productivity $^{1,2}$. Disability following stroke depends on several factors, including the location and extent of brain damage and the speed of assessment and treatment. Approximately $20 \%$ of strokes are fatal, and more than 50\% of those who survive are reliant upon others for the rest of their lives ${ }^{3}$. Around $85 \%$ of stroke patients have an ischaemic stroke where a blood clot (thrombosis) lodges or forms in a brain artery, cutting blood and oxygen supply to a part of the brain ${ }^{4}$. The remaining $15 \%$ of patients experience a haemorrhagic stroke (loss of blood from an artery) although occasionally there is a mixed picture. Early intervention in the case of thrombosis can significantly reduce residual disability and lower mortality (Ibid.). In a large US registry study of 58,353 stroke patients, those who received a clot-busting drug (thrombolysis - such as recombinant tissue plasminogen activator - rt-PA) within 0-90 minutes of stroke symptom onset compared to between 3-4.5 hours were approximately 50\% more likely to walk independently at discharge (adjusted odds ratio (AOR) 1.51, p<0.001) . They were also more likely to be discharged to home (AOR 1.33, p<0.001), and were less likely to die in hospital (AOR: 0.74, $\mathrm{p}<0.001$ ); indeed, every 15 minute delay in symptom onset to treatment time lead to significant deterioration in patient outcome. There is already evidence that treatment of acute stroke is associated with a cost saving in the long-term 6 .

The current gold-standard method of diagnosing stroke type is computed tomography (CT). This scan is essential; treatment cannot be given to patients with a haemorrhagic stroke as it could substantially worsen outcomes. However, CT scanners are large, expensive pieces of equipment that are only available at a limited number of centres of definitive care. It can be difficult to reach, assess and transfer rural stroke patients to a CT scanner within the 4.5 hour critical period for delivering thrombolysis, and is near-impossible to do so within 90 minutes in most cases. In Scotland, the target is to deliver thrombolysis to a minimum of $80 \%$ of eligible stroke patients within one hour of admission to hospital. However, no Scottish hospital meets this target currently (average $=29 \%)^{7}$. Given the time pressure in assessing and treating stroke, and the fact that there is already considerable pressure on CT scanners, there is a growing movement towards doing more for stroke patients before they arrive at hospital $^{8}$.

Transcranial ultrasound is one potential method of generating crucial early aetiological information where access to CT imaging is delayed through remoteness and/or if there is otherwise limited access to CT scanning. However, it is essential that the individual performing the scan remotely - unless an expert in transcranial ultrasound - has access to expert advice in order to facilitate accurate diagnosis. This forms the basis of our 'Satellite Ultrasound for Rural Stroke' project. Images would be streamed directly to an expert who would guide the scanning process and make a diagnosis. In this paper we describe our technological initiative to transmit live ultrasound images via a multi-channel communication device. Although our research programme is driven by a rural need, the concept is equally applicable to urban areas where rapid access to CT scanners can be restricted by a range of operational factors. It is important to stress that the work presented here does not aim to deploy the technology as a clinical intervention for stroke in the immediate future but simply to demonstrate that we can develop an appropriate technological platform. Our research is 
also highly-relevant to a number of ultrasound scans that are already employed widely prehospital; e.g. for screening for pneumothorax and abdominal bleeding.

\section{Transcranial ultrasound as an early screening tool for stroke}

If we could assess stroke aetiology sufficiently accurately before or even during transfer to hospital then such an early screening tool could add value to existing patient management. One method is to bring the gold-standard, in-hospital clinical personnel and technology out into the field. Ebinger et al employed a Stroke Emergency Mobile (STEMO) ambulance, which housed a CT scanner, a point-of-care laboratory, telemedicine link, neurologist, radiology technician and paramedic ${ }^{9}$. Their PHANTOM-S study was conducted in urban Berlin (restricted to a time radius of only 16 minutes from base) and showed that such a service could significantly reduce time to thrombolysis by 25 minutes (95\% CI 20-29 minutes). Also, STEMO resulted in a significantly higher thrombolysis rate, particularly within the first 'golden' hour after symptom onset ${ }^{10}$. Stroke patients treated within the first hour were more likely to be discharged home (AOR: 1.93, p<0.05). However, this model could really only work in a small-radius, urban area with a large number of potential patients; it would not be cost-effective to position such an expensive set of resources in rural locations.

There is already a modest evidence base for the use of portable ultrasound in the immediate, pre-hospital assessment of stroke patients ${ }^{11}$. Groups such as those involved in the International Pre-hospital Stroke Project, have demonstrated that Transcranial Colour-Coded Sonography (TCCS) is a feasible tool for assessing intracranial arteries and can be used to identify occlusion in the middle cerebral arteries ${ }^{12-13}$. Bar et al compared TCCS against CT angiography (CTA) in a single-centre prospective study of 45 patients within three hours of onset of ischaemic stroke ${ }^{14}$. Fourteen patients $(31 \%)$ were excluded, leaving a total of 31 stroke cases. The sensitivity of TCCS to positively identify middle cerebral artery main stem occlusion was $92.3 \%$, and specificity to rule out occlusion was $94.4 \%$. TCCS agreed with the CTA findings in $87.1 \%$ of patients. Allendoerfer et al explored the prognostic value of examining intra- and extracranial arteries using Doppler ultrasound very early on in ischaemic stroke (prospective multi-centre study) ${ }^{15}$. Cerebrovascular ultrasound provided very valuable additional information regarding prognosis, which the authors contended was useful in particular for identifying those patients at high risk of poor outcome.

There is also a limited amount of evidence that transcranial ultrasound in b-mode (2D mode) can be used to identify and rule out haemorrhagic stroke. Mäurer et al demonstrated that ultrasound detected 50/53 (94.3\%) haemorrhages detected by CT, and correctly confirmed the absence of haemorrhage in $76 / 80(95 \%)$ cases $(n=133)$ where brain parenchyma could be visualised $^{16}$. Twelve percent $(n=18)$ of patients had an insufficient bone window to image brain tissue adequately. More recently, Kukulska-Pawluczuk et al showed transcranial ultrasound successfully identified brain haemorrhage in 34/39 cases (sensitivity $87.2 \%$ ) no more than 12 hours after $\mathrm{CT}^{17}$. Approximately twice the proportion of patients $(23.5 \%-\mathrm{n}=12)$ had an insufficient bone window compared with Mäurer's work. Siedel et al reported a lower sensitivity $(78 \%)$ in their study of 23 consecutive patients with brain haemorrhage ${ }^{18}$, and stated that the, "...disadvantages of TCCS compared with CT or MRI are the limited spatial resolution of the ultrasound images and the dropout rate because of insufficient acoustic bone window" (p2065) $)^{19}$. Nevertheless, the value of ultrasound is being able to deliver repeated imaging quickly ${ }^{15}$. Also, most of the studies investigating haemorrhage were conducted some time ago with older ultrasound equipment and with small patient numbers. It is possible, with 
superior modern scanners, plus software - and potentially transducer - refinement, that ultrasound scanning for haemorrhage could be improved.

\section{Technology to access remote expert advice in pre-hospital stroke care}

There is already a considerable body of evidence demonstrating the advantages of 'telestroke' technology-facilitated remote assessment. For example, thrombolysis rates achieved via telestroke have been shown to match rates achieved via on-site expert assessment with comparable patient outcomes ${ }^{20}$. Remote patient assessment generally involves videoconferencing (with telephone as a back-up) and employs a recognised stroke severity scale such as the National Institutes of Health Stroke Scale (NIHSS). A range of telestrokespecific scales are also available, such as the Unassisted TeleStroke Scale (UTSS), which has good correlation with the NIHSS and takes around half the time to complete (3.1 vs. 8.5 minutes, $\mathrm{p}<0.001)^{21}$. Yperzeele et al in their review of pre-hospital stroke care described three generations of telestroke technology ${ }^{8}$, the first two of which saw communication over fixed landline (1.0) and the World Wide Web (2.0) but only for patients who had already arrived at hospital. Telestroke 3.0 moves into the pre-hospital context but only a very limited amount of research has been conducted thus far. Growth in this field of research has been advocated by the American Stroke Association ${ }^{22}$.

Perhaps the most promising pre-hospital telestroke results were reported by a recent healthy volunteer study in Belgium ${ }^{23}$. Forty-one real-life stroke cases were mimicked by two volunteers who were located in an ambulance equipped with state-of-the-art videoconferencing technology. Audio-visual data were streamed 'on the move' to a remote expert via the Internet using a 4G 'ultrabroadband' cellular network. Data were downloaded at a mean speed of 11.0 megabits per second (mbps), but data rate peaked at 20.9 mbps. Telestroke assessments using the UTSS were only suspended once because of technical reasons, and only a minority of screen freezing and poor audio quality were reported. There was also strong correlation between UTSS and NIHSS scores. Nevertheless, this study did not include patients and was conducted using a high data rate communications network; other areas - particularly rural and remote geographies - may not see such technology infrastructure for years to come and so these results have limited transferability. Previous pre-hospital telestroke studies have reported poorer technical performance. For example, TeleBAT experienced issues associated with low bandwidth and communications instability in a moving vehicle, although these data are now reasonably old ${ }^{24}$. A more recent study by Liman et al utilised higher data rate $3 \mathrm{G}$ networks but experienced a $60 \%(18 / 30)$ failure rate due to signal $\operatorname{loss}^{25}$. Telestroke using this particular equipment set-up was not deemed appropriate for clinical use.

Poor connectivity in rural areas of the Scottish Highlands has been shown to be a barrier for electrocardiogram (ECG) transmission from ambulances ${ }^{26}$. The size of an ECG data file is vastly smaller than the size of file, or data stream that would be required for ultrasound imagery. Enhancing bandwidth and making use of several communications channels are critical for facilitating the transmission of high-quality, two-way data, enabling remote expert diagnosis. Other studies have also employed this multiple channel technique for sending medical data ${ }^{27,28}$. 


\section{A technology demonstrator of ultrasound fused with intelligent communication methods}

\section{Project partnership}

This project was developed by a consortium of four key partners:

- The University of Aberdeen Centre for Rural Health (CRH) and Highland Medical Education Centre

- National Health Service clinical staff with expertise in stroke, emergency medicine and the use of ultrasound

- Tactical Wireless Ltd., a small company manufacturing an intelligent, multi-channel communications device

- Health Science Solutions Limited, a specialist ultrasound probe company

The project was also supported by ultrasound manufacturers Philips Healthcare UK and BK Medical (part of the Analogic Ultrasound Group), who provided loan equipment and technical support.

\section{Project aims}

We aimed to link appropriate portable ultrasound machines with an intelligent communications device in order to stream these data via cellular and satellite networks to a remote expert for real-time review. We also planned to evaluate the quality of data (ultrasound and audio-visual data) sent via the device using a field-function methodology.

\section{Description of the intelligent communications device}

The Omni-Hub ${ }^{\mathrm{TM}}$, developed by Tactical Wireless Ltd. (parent company Morton Manufacturing Ltd., Aylesbury, Bucks., UK) is a universal sensor and communications hub which is capable of generating its own long-range Wi-Fi hotspot, to take inputs from a range of digital IP devices, including a wide variety of medical and non-medical devices. Omni$\mathrm{Hub}^{\mathrm{TM}}$ is Windows-based (Microsoft, Redmond, WA) and has a state-of-the-art video management system that can store and forward device output. Videos are stored as a series of watermarked JPEGs and the rate of live streaming video transmission can be adjusted to suit available bandwidth. The recorded watermarked JPEGs are recombined at the receiving centre, to reconstitute the video. Omni-Hub ${ }^{\mathrm{TM}}$ has an intelligent backhaul router, which can simultaneously utilise multiple cellular networks, satellite networks, Wi-Fi and ADSL. The routers all go through the Omni-Hub.net facility which can manage multiple Hubs, enabling substantial bandwidth to be available in a major emergency, by aggregating the bandwidth of each Omni-Hub ${ }^{\mathrm{TM}}$. Apps, for secure video conferencing and the conversion of a smartphone to a secure push-to-talk walkie-talkie device, have been integrated. The system's open architecture enables any device to be integrated. The system also provides a high level of security: communication between digital IP devices uses a virtual private network; the video management system recombines the JPEGs at the destination; the system is capable of a 256 Advanced Encryption Standard (with the option of adding military encryption, if required) and the use of multiple backhaul routes provides a high level of cyber security. The data feeds are currently viewed using the secure Crossfire ${ }^{\mathrm{TM}}$ graphical user interface (VayTek Inc., Fairfield, IA) through which pixels and frames-per-second can be altered in real-time for each channel. More than 2,000 copies of the embedded video management system have been delivered to US Federal Agencies. The rack-mounted version of Omni-Hub ${ }^{\text {TM }}(400 \times 300 \times 89$ 
$\mathrm{mm} ; 4.1 \mathrm{Kg}$ ) would run off an internal ambulance power source, although a portable, batterypowered system with four hours of endurance is also under development.

\section{Ultrasound operators}

We propose that ambulance clinicians would be the most likely operators of transcranial ultrasound pre-hospital. There is a precedent from another condition: the Scottish Ambulance Service and general practitioners administer thrombolysis for acute myocardial infarction following advice from coronary care staff assessing ECGs transmitted from ambulances or primary care settings ${ }^{26}$. As such, similar treatment pathways and protocols are already in place for other conditions in potentially life-saving emergencies. There is already good evidence that ambulance paramedics can generate diagnostic-quality ultrasound images with remote support. For example, Boniface et al demonstrated that 51 paramedics with no prior ultrasound experience and only 20 minutes of training completed the Focussed Assessment with Sonography in Trauma (FAST) suite of scans very successfully and quickly $(<5$ minutes) under remote direction from an Emergency Physician ${ }^{29}$. However, this was essentially a laboratory-type, room-to-room study and so did not replicate what might happen in real-life. These data do not mean that paramedics could generate diagnostic quality images of the brain using ultrasound, but do deliver a successful precedent for the type of novice ultrasound end-user we propose.

Description of the ultrasound communication method and possible clinical protocol for acute stroke (see Figure)

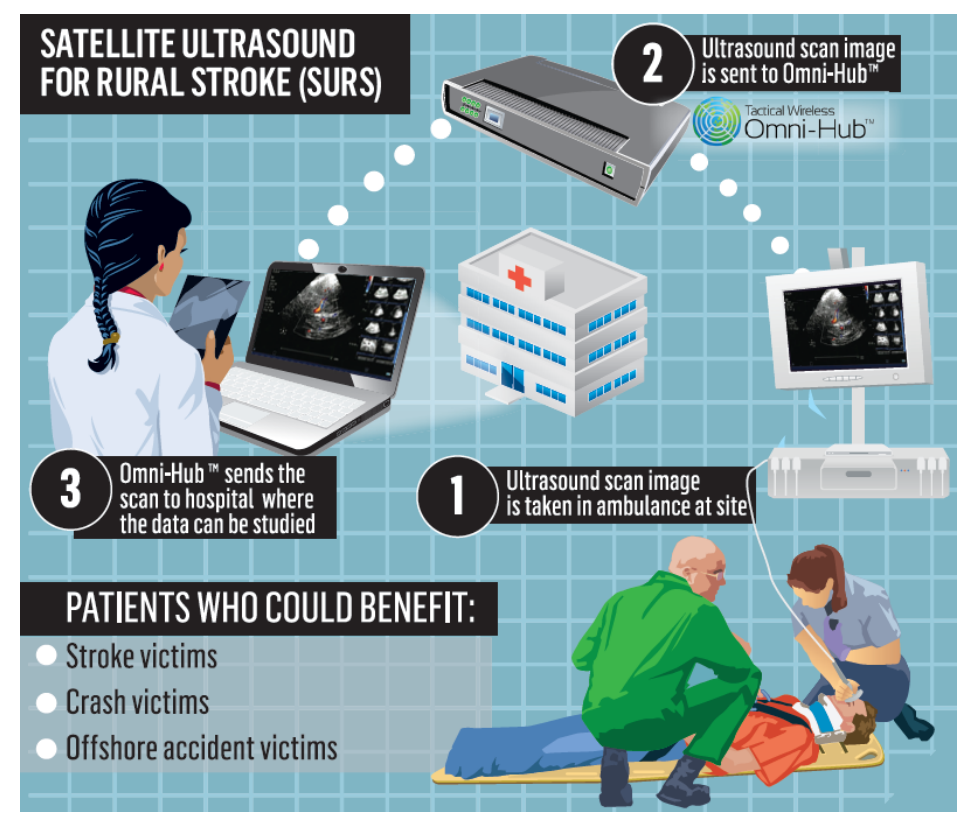

Figure. Proposed model of technology use

(permission to reproduce image granted by the Press and Journal newspaper)

i. Ambulance clinicians/first responders arrive on scene and conduct an initial primary survey of patient airway, breathing and circulation. After implementing any required life-saving management, personnel would then establish the clinical likelihood of stroke using the Face Arm Speech Test ${ }^{30}$. Ambulance paramedics show good agreement of neurological deficits with physicians when using this tool $^{31}$. If positive, the patient is considered for remotely-supported transcranial 
ultrasound investigation and a remote expert is alerted (although it is important to note that not all positive scoring patients will have had a stroke - this emphasises the importance of being seen by a stroke specialist).

ii. The ultrasound operator would explore for the presence of intracranial haemorrhage using b-mode ultrasound imaging. This would be done through transtemporal acoustic windows on either side of the head. Other 'windows' into the brain could also be explored, such as transforaminal imaging at the rear of the head, giving greater confidence that every part of the brain has been scanned. Ultrasound video and images would be streamed in real-time for review by the remote expert, along-with head-cam video showing the orientation of the ultrasound transducer, and two-way voice communication. The default position will be to maximise the number and consistency of frames per second whilst retaining sufficient quality and resolution of ultrasound images.

iii. Communications coverage will vary for a variety of reasons. For example, very remote and rural areas may only have satellite coverage, which requires line-ofsight with a geostationary satellite. The current generation of satellites lie near to the horizon to the south in the Highlands of Scotland, which means that satellite communication could be impeded when mountains also lie to the south. Closer to urban centres there will be cellular coverage, ranging from $2 \mathrm{G}$ to $4 \mathrm{G}$, although in the Highlands of Scotland 3G coverage is sparse and when present is the best available. Cellular coverage will vary according to distance from the nearest basestation and geographical formations that may impede signal. The Omni-Hub ${ }^{\mathrm{TM}}$ can also be configured to minimise data costs; satellite communications have reduced in cost, but are still considerably more expensive than cellular networks. In the proposed clinical application, satellite communications would only be employed when cellular bandwidth is insufficient for transmitting images of sufficient quality for remote diagnosis.

iv. If the patient has an adequate transtemporal bone window to conduct a transcranial ultrasound scan (i.e. so that the operator can see key anatomical markers such as the margins of the cranial vault on the opposite side of the head, for example - around $15 \%$ of patients may not have an adequate window ${ }^{32}$ ), and if the scan rules out intracranial haemorrhage, then this could open up the opportunity for pre-hospital thrombolysis (combined with the traditional clinical assessment). This will only be considered if a subsequent, large-scale, concurrent validity study of CT vs. ultrasound demonstrates that ultrasound is adequately sensitive and specific in ruling out intracranial haemorrhage. However, any reduction in sensitivity and specificity would need to be matched with improved outcomes resulting from rapid treatment.

\section{Preliminary results}

Two ultrasound machines (Philips CX50, Philips Healthcare, Andover, MA; Ultrasonix Sonix Tablet, Analogic Ultrasound, Peabody MA) were linked with the Omni-Hub ${ }^{\mathrm{TM}}$ and video sent successfully to a remote tablet computer using cellular networks with around 1 second latency. This proved the feasibility of using the Omni-Hub ${ }^{\mathrm{TM}}$ to send ultrasound video in real- 
time using wireless communication networks. We commenced our experimental runs proper by conducting telestroke assessments ${ }^{33}$. This served as a suitable test of the system prior to transmitting higher-bandwidth ultrasound data. Twelve healthy volunteers were recruited to the study and acted as a patient and/or paramedic. Participants read a variety of scripts that described 1) patients with a non-stroke condition, 2) those with contraindications to thrombolysis, and 3) stroke patients who were candidates for thrombolysis. Audio-visual data were transmitted using a combination of $2 \mathrm{G}$ and $3 \mathrm{G}$ networks from 15 locations around Inverness to a mock clinical control centre staffed by participating physicians. A total of 23 telestroke assessments were completed; 19 while the vehicle was in motion and 4 while stationary. Mean data upload rate was 1,250 kilobits per second (Kbps) but ranged widely from 22-1,900 kbps. Mean latency was 0.3 seconds. The mean data upload rate associated with a higher audio-visual quality rating (i.e. $4 / 5$ or $5 / 5$ ) was $1,021 \mathrm{Kbps}$.

We are currently analysing data from the ultrasound transmission part of the study, which took place at the same time as the telestroke assessments and used the same healthy volunteers. Participants 1) acted as novice scanners, and 2) if they were willing, also acted as mock patients to be scanned by other novice scanners. The ultrasound scans included a mix of known and unknown scans. The known scans comprised several of the FAST suite of assessments, and the unknown scan was a transcranial image of the brain, focused on identifying the position of the third ventricle. The transcranial scan was undertaken using a cardiac transducer through transtemporal acoustic windows on either side of the head. Scanning commenced in the vertical plane and image 'depth' was increased in order to identify the opposite side of the cranial vault. The transducer was rotated as required in order to capture the best possible image of the brain (as the size and position of the transtemporal window is known to vary). An attempt was then made to identify the position of the third ventricle. The mix of known and unknown scans was intentional as the transcranial scan was generally not well known and as such not used routinely by physicians. Also, the focus of the investigation was on ultrasound image quality in general, not transcranial imaging alone. Participants (none of whom had any prior experience with ultrasound scanning) received a group one-hour training session from an emergency physician trained in point-of-care ultrasound. Ultrasound imaging took place in a research ambulance and the data transmitted via cable to an Omni-Hub ${ }^{\mathrm{TM}}$ mounted in a separate vehicle alongside; ultrasound data capture and transmission took place while stationary. Telemedicine audio and visual feeds ran concurrently with ultrasound data transmission.

\section{Potential opportunities and challenges}

Our prototype technological solution has the potential to facilitate the early assessment of patients who have had a suspected stroke. In its simplest form this could involve a telestroke assessment, carried out in the ambulance en-route to hospital with a live video link to a remote expert. This would not involve any delay, and could potentially minimise the time to receive a CT scan. However, the staffing of such an initiative could be complex as it would require the availability of remote stroke clinicians at short notice, 24 hours a day. Nevertheless, this model has already been implemented in an international acute telestroke initiative between Scotland and New Zealand; UK clinicians provide thrombolysis decision support to a New Zealand hospital during their out-of-hours time ${ }^{34}$.

There are also considerable clinical and technical challenges to pre-hospital, paramedicoperated transcranial ultrasound becoming a service: 
- Using transcranial ultrasound to detect stroke is by no means simple: while there is significant evidence for Doppler ultrasound being diagnostic for ischaemic stroke, the evidence for use of b-mode ultrasound to detect haemorrhagic stroke is as yet inadequate for clinical deployment;

- Transcranial ultrasound cannot produce viable images in some people who do not have a suitable transtemporal bone window. It is possible that the use of alternative windows or lower frequency transducers with smaller footprints - or microbubble contrast - could help with the imaging of these patients;

- Ultrasound cannot detect brain lesions (e.g. tumours) that are causing stroke-like symptoms, whereas CT can;

- Ultimately, a large, hospital-based (i.e. under ideal conditions) concurrent validity study of ultrasound vs. CT would be necessary. As a pre-cursor to this it may also be necessary to refine algorithms within ultrasound equipment, enhancing the imaging of free blood in the brain;

- Pre-hospital, non-radiologist clinicians would require training in the technique of scanning the brain, and remote experts would need training to interpret the ultrasound images;

- The technical aspects of sending ultrasound images would require to be integrated within NHS IT systems. However, image delivery from remote locations would be relatively simple compared with the clinical challenges.

Clearly, we are at the beginning of a new mode of patient assessment, but one that could deliver significant patient benefit in the future. Others are also evaluating novel technologies for establishing the aetiology of stroke pre-hospital. For example, Persson et al have recently reported positive results using microwaves delivered through a helmet assembly ${ }^{35}$. This is an emerging area of research with potential long-term promise.

\section{Future work}

There are two main avenues for future work once the communications technology is established. The first will be a concurrent validity study comparing ultrasound with CT in order to demonstrate whether ultrasound is adequate for establishing stroke aetiology in the pre-hospital environment. Secondly, the potential utility of remote ultrasound diagnosis with non-expert operators guided by experts in specialist centres should be explored for the most remote healthcare settings such as the oil and gas exploration sector, including on- and offshore installations. The economic benefits of such an approach may be substantial given that emergency evacuations from remote installations are highly costly: it may be possible to achieve adequate diagnostic precision in commonly suspected conditions such as acute appendicitis to allow better decisions on whether to transfer or manage cases in situ.

\section{Acknowledgement:}

This research was funded by Highlands \& Islands Enterprise, the UK Technology Strategy Board's Space and Life Sciences Catapult, the University of Aberdeen's dot.rural Digital Economy Hub and by TAQA Bratani. 


\section{References}

1. Townsend N, Wickramasinghe K, Bhatnagar P, Smolina K, Nichols M, Leal J et al. Coronary heart disease statistics 2012 edition. London: British Heart Foundation 2012.

2. Department of Health National Audit Office. Progress in improving stroke care London: The Stationary Office 2010.

3. Royal College of Physicians National Sentinel Stroke Clinical Audit 2010 Round 7 Public report for England, Wales and Northern Ireland. Prepared on behalf of the Intercollegiate Stroke Working Party May 2011.

4. Intercollegiate Stroke Working Party. National clinical guideline for stroke, 4th edition. London: Royal College of Physicians 2012.

5. Saver JL, Fonarow GC, Smith EE, Reeves MJ, Grau-Sepulveda MV, Pan W et al. Time to Treatment With Intravenous Tissue Plasminogen Activator and Outcome From Acute Ischaemic Stroke. Journal of the American Medical Association 2013;309(23):2480-2488.

6. Jung K-T, Shin DW, Lee K-J, Oh M. Cost-Effectiveness of Recombinant Tissue Plasminogen Activator in the Management of Acute Ischaemic Stroke: A Systematic Review. Journal of Clinical Neurology 2010;6(3):117-126.

7. Information Services Division Scotland. Scottish Stroke Care Audit: 2013 National Report. NHS National Services Scotland: Edinburgh.

8. Yperzeele L, Van Hooff R-J, Smedt AD, Espinoza AV, Van De Casseye R, Hubloue I et al. Prehospital Stroke Care: Limitations of Current Interventions and Focus on New Developments. Cerebrovascular Diseases 2014;38(1):1-9.

9. Ebinger M, Winter B, Wendt M, Weber J, Waldschmidt C, Rozanski $M$ et al. Effect of the Use of Ambulance-Based Thrombolysis on Time to Thrombolysis in Acute Ischaemic Stroke: A Randomized Clinical Trial. Journal of the American Medical Association 2014;311(16):1622-1631.

10. Ebinger M, Kunz A, Wendt M, Rozanski M, Winter B, Waldschmidt C et al. Effects of Golden Hour Thrombolysis: A Prehospital Acute Neurological Treatment and Optimization of Medical Care in Stroke (PHANTOM-S) Substudy. JAMA Neurology 2014;Published online $17^{\text {th }}$ November 2014

11. Hölscher T. Prehospital use of portable ultrasound for stroke diagnosis and treatment initiation. Air Rescue 2012;5(2):64-67.

12. Hölscher T, Schlachetzki F, Zimmermann M, Jakob W, Ittner KP, Haslberger J et al. Transcranial ultrasound from diagnosis to early stroke treatment. 1. Feasibility of prehospital cerebrovascular assessment. Cerebrovascular Diseases 2008;26(6):659-663.

13. Schlachetzki F, Herzberg M, Hölscher T, Ertl M, Zimmermann M, Ittner KP et al. Transcranial ultrasound from diagnosis to early stroke treatment - Part 2: prehospital neurosonography in patients with acute stroke - The Regensburg stroke mobile project. Cerebrovascular Diseases 2012;33(3):262-271.

14. Bar M, Školoudik D, Roubec M, Hradilek P, Chmelova J, Czerny D et al. Transcranial Duplex Sonography and CT Angiography in Acute Stroke Patients. Journal of Neuroimaging 2010;20(3):240-245.

15. Allendoerfer J, Goertler M \& von Reutern G-M. Prognostic relevance of ultraearly doppler sonography in acute ischaemic stroke: a prospective multicentre study. Lancet Neurology 2006;5(10):835-840. 
16. Mäurer M, Shambal S, Berg D, Woydt M, Hofmann E, Georgiadis D et al. Differentiation between intracerebral hemorrhage and ischemic stroke by transcranial color-coded duplex-sonography. Stroke 1998;29(12):2563-2567.

17. Kukulska-Pawluczuk B, Książkiewicz B \& Nowaczewska M. Imaging of spontaneous intracerebral hemorrhages by means of transcranial color-coded sonography. European Journal of Radiology 2011;81(6):1253-1258.

18. Siedel G, Kaps M \& Dorndorf W. Transcranial color-coded duplex sonography of intracerebral hematomas in adults. Stroke 1993;24(10):1519-1527.

19. Siedel G, Kaps M \& Gerriets T. Potential and limitations of transcranial colorcoded sonography in stroke patients. Stroke 1995;26(11):2061-2066.

20. Sairanen T \& Tatlisumak T. Finnish telestroke: An overview. European Research in Telemedicine 2012;1(3-4):115-117.

21. Van Hooff R-J, De Smedt A, De Raedt S, Moens M, Mariën P, Paquier P et al. Unassisted Assessment of Stroke Severity Using Telemedicine. Stroke 2013;44(5):1249-1255.

22. Schwamm LH, Audebert HJ, Amarenco P, Chumbler NR, Frankel MR, George MG et al. Recommendations for the Implementation of Telemedicine Within Stroke Symptoms of Care: A Policy Statement From the American Heart Association. Stroke 2009;40(7):2635-2660.

23. Van Hooff R-J, Cambron M, Van Dyck R, De Smedt A, Moens M, Espinoza AV et al. Prehospital Unassisted Assessment of Stroke Severity Using Telemedicine: A Feasibility Study. Stroke 2013;44(10):2907-2909.

24. LaMonte MP, Xiao Y, Hu PF, Gagliano DM, Bahouth MN, Gunawardane RD et al. Shortening Time to Stroke Treatment Using Ambulance Telemedicine: TeleBAT. Journal of Stroke and Cerebrovascular Diseases 2004;13(4):148-154.

25. Liman TG, Winter B, Waldschmidt C, Zerbe N, Hufnagl P, Audebert HJ et al. Telestroke Ambulances in Prehospital Stroke Management: Concept and Pilot Feasibility Study. Stroke 2012;43(8):2086-2090.

26. Rushworth GF, Bloe C, Lesley Diack H, Reilly R, Murray C, Stewart D et al. PreHospital ECG E-Transmission for Patients with Suspected Myocardial Infarction in the Highlands of Scotland. International Journal of Environmental Research and Public Health 2014;11:2346-2360.

27. Bergrath S, Czaplik M, Rossaint R, Hirsch F, Beckers SK, Valentin B et al. Implementation phase of a multicentre prehospital telemedicine system to support paramedics: Feasibility and possible limitations. Scandinavian Journal of Trauma, Resuscitation and Emergency Medicine 2013;21(1):article number 54.

28. Czaplik M, Bergrath S, Rossaint R, Thelen S, Brodziak T, Valentin B et al. Employment of Telemedicine in Emergency Medicine. Methods of Information in Medicine 2014;53(2):99-107.

29. Boniface KS, Shokoohi H, Smith ER \& Scantlebury K. Tele-ultrasound and paramedics: real-time remote physician guidance of the Focused Assessment With Sonography for Trauma examination. American Journal of Emergency Medicine 2011;29(5):477-481.

30. Harbison J, Hossain O, Jenkinson D, Davis J, Louw SJ, Ford GA. Diagnostic Accuracy of Stroke Referrals From Primary Care, Emergency Room Physicians, and Ambulance Staff Using the Face Arm Speech Test. Stroke 2003;34(1):71-76.

31. Mohd Nor A, McAllister C, Louw SJ, Dyker AG, Davis M, Jenkinson D et al. Agreement Between Ambulance Paramedic- and Physician-Recorded Neurological Signs with Face Arm Speech test (FAST) in Acute Stroke Patients. Stroke 2004;35(6):1355-1359. 
32. Meyer-Wiethe K, Sallustio F, Kern R. Diagnosis of Intracerebral Hemorrhage with Transcranial Ultrasound. Cerebrovascular Diseases 2009;27(suppl 2):40-47.

33. Eadie L, Regan L, Mort A, Shannon H, Walker J, MacAden A et al. Telestroke Assessment on the Move: Prehospital Streamlining of Patient Pathways. Stroke 2014; online first December 302014.

34. Ranta A, Whitehead M, Gunawardana C \& Reoch A. Feasibility of an international telestroke service: Initial treatment outcomes. Journal of Clinical Neuroscience 2014;21(11):2040-2041.

35. Persson M, Fhager A, Trefna H, Yu Y, McKelvey $T$, Pegenius $G$ et al. Microwave-based stroke diagnosis making global pre-hospital thrombolytic treatment possible. IEEE Transactions on Biomedical Engineering 2014;99. 